\title{
PROPERTIES OF THE FIBER CONE OF IDEALS IN LOCAL RINGS
}

\author{
William J. Heinzer \\ Department of Mathematics, Purdue University \\ West Lafayette, Indiana 47907 USA \\ E-mail: heinzer@math.purdue.edu \\ Mee-Kyoung Kim \\ Department of Mathematics, Sungkyunkwan University \\ Jangangu Suwon 440-746, Korea \\ E-mail: mkkim@math.skku.ac.kr
}

\begin{abstract}
For an ideal $I$ of a Noetherian local ring $(R, \mathbf{m})$ we consider properties of $I$ and its powers as reflected in the fiber cone $F(I)$ of $I$. In particular, we examine behavior of the fiber cone under homomorphic image $R \rightarrow R / J=R^{\prime}$ as related to analytic spread and generators for the kernel of the induced map on fiber cones $\psi_{J}: F_{R}(I) \rightarrow F_{R^{\prime}}\left(I R^{\prime}\right)$. We consider the structure of fiber cones $F(I)$ for which $\operatorname{ker} \psi_{J} \neq 0$ for each nonzero ideal $J$ of $R$. If $\operatorname{dim} F(I)=d>0$, $\mu(I)=d+1$ and there exists a minimal reduction $J$ of $I$ generated by a regular sequence, we prove that if $\operatorname{grade}\left(G_{+}(I)\right) \geq d-1$, then $F(I)$ is Cohen-Macaulay and thus a hypersurface.
\end{abstract}

\section{INTRODUCTION}

For an ideal $I$ in a Noetherian local ring $(R, \mathbf{m})$, the fiber cone of $I$ is the graded ring

$$
F(I)=\bigoplus_{n \geq 0} F_{n}=\bigoplus_{n \geq 0} I^{n} / \mathbf{m} I^{n} \cong R[I t] / \mathbf{m} R[I t],
$$

where $R[I t]$ is the Rees ring of $I$ and $F_{n}=I^{n} / \mathbf{m} I^{n}$. We sometimes write $F_{R}(I)$ to indicate we are considering the fiber cone of the ideal $I$ of the ring $R$. In terms of the height, $\operatorname{ht}(I)$, of $I$ and the dimension, $\operatorname{dim} R$, of $R$, one always has the inequalities $\operatorname{ht}(I) \leq \operatorname{dim} F(I) \leq \operatorname{dim} R$.

For an arbitrary ideal $I \subseteq \mathbf{m}$ of $(R, \mathbf{m})$, the fiber cone $F(I)$ has the attractive property of being a finitely generated graded ring over the residue field $k:=R / \mathbf{m}$

Date: January 9, 2003.

1991 Mathematics Subject Classification. Primary: 13A30, 13C05; Secondary: 13E05, $13 \mathrm{H} 15$.

Key words and phrases. fiber cone, analytic spread, multiplicity, Cohen-Macaulay ring.

We thank Bernd Ulrich for helpful conversations about the topics considered here.

The second author is supported by Korea Research Foundation Grant (KRF-2000-015-DP0005). 
that is generated in degree one, i.e., $F_{n}=F_{1}^{n}$ for each positive integer $n$, so $F(I)=$ $k\left[F_{1}\right]$.

It is well known in this setting that the Hilbert function $H_{F}(n)$ giving the dimension of $I^{n} / \mathbf{m} I^{n}$ as a vector space over $k$ is defined for $n$ sufficiently large by a polynomial $h_{F}(X) \in \mathbb{Q}[X]$, the Hilbert polynomial of $F(I)$ [Mat, Corollary, page 95], [AM, Corollary 11.2]. A simple application of Nakayama's lemma, [Mat, Theorem 2.2 ], shows that the cardinality of a minimal set of generators of $I^{n}, \mu\left(I^{n}\right)$, is equal to $\lambda\left(I^{n} / \mathbf{m} I^{n}\right)$, the value of the Hilbert function $H_{F}(n)$ of $F(I)$.

An interesting invariant of the ideal $I$ is its analytic spread, denoted $\ell(I)$, where the analytic spread of $I$ is by definition the dimension of the fiber cone, $\ell(I)=$ $\operatorname{dim} F(I)[\mathrm{NR}]$. The analytic spread measures the asymptotic growth of the minimal number of generators of $I^{n}$ as a function of $n$. In relation to the degree of the Hilbert polynomial, we have the equality $\ell(I)=1+\operatorname{deg} h_{F}(X)$. An ideal $J \subseteq I$ is said to be a reduction of $I$ if there exists a positive integer $n$ such that $J I^{n}=I^{n+1}$. It then follows that $J^{i} I^{n}=I^{n+i}$ for every postive integer $i$. If $J$ is a reduction of $I$, then $J$ requires at least $\ell(I)$ generators. If the residue field $R / \mathbf{m}$ is infinite, then minimal reductions of $I$ correspond to Noether normalizations of $F(I)$ in the sense that $a_{1}, \ldots, a_{r} \in I-I^{2}$ generate a minimal reduction of $I$ if and only if their images $\overline{a_{i}} \in I / \mathbf{m} I \subseteq F(I)$ are algebraically independent over $R / \mathbf{m}$ and $F(I)$ is integral over the polynomial ring $(R / \mathbf{m})\left[\overline{a_{1}}, \ldots, \overline{a_{r}}\right]$. In particular, if $R / \mathbf{m}$ is infinite, then there exist $\ell(I)$-generated reductions of $I$,

For a positive integer $s$, the fiber cone $F\left(I^{s}\right)$ of the ideal $I^{s}$ embeds in the fiber cone $F(I)=\oplus_{n=0}^{\infty} F_{n}$ of $I$ by means of $F\left(I^{s}\right) \cong \oplus_{n=0}^{\infty} F_{n s}$. This isomorphism makes $F(I)$ a finitely generated integral extension of $F\left(I^{s}\right)$. Thus $\operatorname{dim} F(I)=\operatorname{dim} F\left(I^{s}\right)$ and $\ell(I)=\ell\left(I^{s}\right)$.

We are particularly interested in conditions that imply the fiber cone $F(I)$ is a hypersurface. Suppose $\operatorname{dim} F(I)=d>0$ and $\mu(I)=d+1$. If $I$ has a reduction generated by a regular sequence and if grade $\left(G_{+}(I)\right) \geq d-1$, we prove in Theorem 5.6 that $F(I)$ is a hypersurface. We have learned from Bernd Ulrich that this result also follows from results in the paper [CGPU] of Corso-Ghezzi-Polini-Ulrich.

A useful property of the analytic spread $\ell(I)$ is that it gives an upper bound on the number of elements needed to generate $I$ up to radical. This property of generation up to radical behaves well with respect to analytic spread of a homomorphic image in the following sense:

Lemma 1.1. Suppose $I \subseteq \mathbf{m}$ is an ideal of a Noetherian local ring $(R, \mathbf{m})$, where $R / \mathbf{m}$ is infinite. Let $a \in I$ and let $R^{\prime}:=R / a R$ and $I^{\prime}:=I R^{\prime}$. If $a_{1}^{\prime}, \ldots, a_{s}^{\prime} \in R^{\prime}$ 
are such that $\operatorname{rad}\left(a_{1}^{\prime}, \ldots, a_{s}^{\prime}\right) R^{\prime}=\operatorname{rad} I^{\prime}$ and if $a_{i} \in R$ is a preimage of $a_{i}^{\prime}$, then $\operatorname{rad} I=\operatorname{rad}\left(a_{1}, \ldots, a_{s}, a\right) R$. In particular, if $\ell\left(I^{\prime}\right)=s$, then $I$ can be generated up to radical by $s+1$ elements.

Proof. Assume that $\operatorname{rad}\left(a_{1}^{\prime}, \ldots, a_{s}^{\prime}\right) R^{\prime}=\operatorname{rad} I^{\prime}$. If $x \in \operatorname{rad} I$, then for some positive integer $n$, we have $x^{n}=y \in I$. Hence the image $y^{\prime}$ of $y$ in $R^{\prime}$ is in $\operatorname{rad}\left(a_{1}^{\prime}, \ldots, a_{s}^{\prime}\right) R^{\prime}$. Therefore $y$ and hence also $x$ is in $\operatorname{rad}\left(a_{1}, \ldots, a_{s}, a\right) R$.

Examples given by Huckaba in [Hu, Examples 3.1 and 3.2] establish the surprising fact of the existence of 3-generated height-2 prime ideals $I$ of a 3-dimensional regular local ring $R$ for which $\operatorname{dim} F(I)=3=\operatorname{dim} R$ and for which there exists a principal ideal $J=x R \subseteq I$ such that if $R^{\prime}:=R / x R$ and $I^{\prime}:=I R^{\prime}$, then $\operatorname{dim} F_{R^{\prime}}\left(I^{\prime}\right)=1<$ $\operatorname{dim} R^{\prime}=\operatorname{dim} R-1$. This result of Huckaba shows that a statement analogous to Lemma 1.1 for reductions, rather than generators up to radical, is false, that is, it is possible that $I^{\prime}=I / a R$ has an $s$-generated reduction while every reduction of $I$ requires at least $s+2$ generators.

These interesting examples are the original motivation for our interest in the behavior of analytic spread in a homomorphic image.

\section{BeHAVIOR OF THE FIBER CONE UNDER HOMOMORPHIC IMAGE.}

Setting 2.1. Let $J \subseteq \mathbf{m}$ be an ideal of a Noetherian local ring $(R, \mathbf{m})$, let $R^{\prime}:=R / J$, and let $\mathbf{m}^{\prime}=\mathbf{m} / J$. For an ideal $I \subseteq \mathbf{m}$ of $R$ let $I^{\prime}=(I+J) / J=I R^{\prime}$ denote the image of $I$ in $R^{\prime}$. There is a canonical surjective ring homomorphism of the fiber cone $F_{R}(I)$ of $I$ onto the fiber cone $F_{R^{\prime}}\left(I^{\prime}\right)$.

We have $R[I t]=\bigoplus_{n \geq 0} I^{n} t^{n}$ and $R^{\prime}\left[I^{\prime} t\right]=\bigoplus_{n \geq 0}\left(I^{\prime}\right)^{n} t^{n}$. Since

$$
\left(I^{\prime}\right)^{n}=\left(I^{n}+J\right) / J \cong I^{n} /\left(I^{n} \cap J\right),
$$

there is a canonical surjective homomorphism of graded rings $\phi_{J}: R[I t] \rightarrow R^{\prime}\left[I^{\prime} t\right]$, with ker $\phi_{J}=\bigoplus_{n \geq 0}\left(I^{n} \cap J\right) t^{n}$.

Since $F_{R}(I)=R[I t] / \mathbf{m} R[I t]$ and $F_{R^{\prime}}\left(I^{\prime}\right)=R^{\prime}\left[I^{\prime} t\right] / \mathbf{m}^{\prime} R^{\prime}\left[I^{\prime} t\right]$, the homomorphism $\phi_{J}: R[I t] \rightarrow R^{\prime}\left[I^{\prime} t\right]$ induces a surjective homomorphism $\psi_{J}: F_{R}(I) \rightarrow F_{R^{\prime}}\left(I^{\prime}\right)$ which preserves grading. This is displayed in the following commutative diagram for which the rows are exact and the column maps are surjective: 


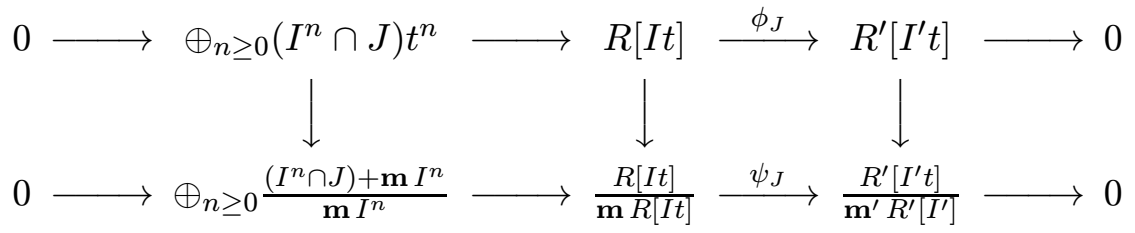

Since we are interested in the behavior of the fiber cone under homomorphic image, we are especially interested in

$$
\operatorname{ker} \psi_{J}=\oplus_{n \geq 0} \frac{\left(I^{n} \cap J\right)+\mathbf{m} I^{n}}{\mathbf{m} I^{n}}
$$

Remark 2.2. Let $(R, \mathbf{m})$ be a Noetherian local ring and let $I \subseteq \mathbf{m}$ be an ideal of $R$. Suppose $J_{1} \subseteq J_{2} \subseteq \mathbf{m}$ are ideals of $R$. Let $R_{i}:=R / J_{i}, i=1,2$, and let $\psi_{i}: F_{R}(I) \rightarrow F_{R_{i}}\left(I R_{i}\right)$ denote the canonical surjective homomorphisms on fiber cones as in (2.1). Then $R_{2} \cong R_{1} / J^{\prime}$, where $J^{\prime}=J_{2} / J_{1}$, and there exists a canonical surjective homomorphism $\psi^{\prime}: F_{R_{1}}\left(I R_{1}\right) \rightarrow F_{R_{2}}\left(I R_{2}\right)$ such that $\psi_{2}=\psi^{\prime} \circ \psi_{1}$.

With notation as in (2.1), if $J$ is a nilpotent ideal of $R$, then $\operatorname{ker} \psi_{J}$ is a nilpotent ideal of $F_{R}(I)$. For suppose $x \in J$ is such that $x^{s}=0$. If $\bar{x} \in \frac{\left(I^{n} \cap J\right)+\mathbf{m} I^{n}}{\mathbf{m} I^{n}}=F_{n}$ is the image of $x$ in $F(I)$, then by definition $\bar{x}^{s}$ is the image of $x^{s}$ in $F_{s n}$, so $\bar{x}^{s}=0$. Thus for $J$ a nilpotent ideal of $R$, we have $\operatorname{dim} F_{R}(I)=\operatorname{dim} F_{R^{\prime}}\left(I^{\prime}\right)$ and $\ell(I)=\ell\left(I^{\prime}\right)$.

Applying this to the situation considered in (2.2), if $s$ is a positive integer, $J_{1}=J^{s}$ and $J_{2}=J$, then with $\psi^{\prime}: F_{R_{1}}\left(I R_{1}\right) \rightarrow F_{R_{2}}\left(I R_{2}\right)$ as in $(2.2)$, it follows that ker $\psi^{\prime}$ is a nilpotent ideal and in this situation $\operatorname{dim} F_{R_{1}}\left(I R_{1}\right)=\operatorname{dim} F_{R_{2}}\left(I R_{2}\right)$. In particular for the examples of Huckaba [Hu, Examples 3.1 and 3.2] mentioned in the end of Section 1, going modulo a power $x^{n} R$ of the ideal $x R$ also reduces the dimension of the fiber cone $F(I)$ from 3 to 1 .

Proposition 2.3. With notation as in Setting 2.1, we have the following implications of Remark 2.2.

(1) If $J^{\prime} \subseteq J$ are ideals of $R$ and if $\operatorname{ker} \psi_{J}=0$, then $\operatorname{ker} \psi_{J^{\prime}}=0$.

(2) $\operatorname{ker} \psi_{J}=0$ if and only if $\operatorname{ker} \psi_{x R}=0$ for each $x \in J$.

(3) For $x \in \mathbf{m}$, we have $\operatorname{ker} \psi_{x R}=0$ if and only if $\left(I^{n}: x\right)=\left(\mathbf{m} I^{n}: x\right)$ for each $n \geq 0$.

Proof. Statements (1) and (2) are clear in view of (2.2) and the description of ker $\psi_{J}$ given in (2.1). For statement (3), we use that $I^{n} \cap x R=x\left(I^{n}: x\right)$. Thus $0=\operatorname{ker} \psi_{x R}=\oplus_{n \geq 0} \frac{\left(I^{n} \cap x R\right)+\mathbf{m} I^{n}}{\mathbf{m} I^{n}} \Longleftrightarrow\left(I^{n} \cap x R\right) \subseteq \mathbf{m} I^{n}$ for each $n \Longleftrightarrow x\left(I^{n}\right.$ : $x) \subseteq \mathbf{m} I^{n}$ for each $n \Longleftrightarrow\left(I^{n}: x\right) \subseteq\left(\mathbf{m} I^{n}: x\right)$ for each $n$. This last statement is equivalent to $\left(I^{n}: x\right)=\left(\mathbf{m} I^{n}: x\right)$ for each $n$. 
Proposition 2.4. Let $(R, \mathbf{m})$ be a Noetherian local ring and let $I \subseteq \mathbf{m}$ be an ideal of $R$. Suppose $J_{1}$ and $J_{2}$ are ideals of $R$ such that $\operatorname{rad} J_{1}=\operatorname{rad} J_{2}$. Let $R_{i}:=R / J_{i}, i=1,2$, and let $\psi_{i}: F_{R}(I) \rightarrow F_{R_{i}}\left(I R_{i}\right)$ denote the canonical surjective homomorphisms on fiber cones as in (2.1). Then $\operatorname{dim} F_{R_{1}}\left(I R_{1}\right)=\operatorname{dim} F_{R_{2}}\left(I R_{2}\right)$ and $\ell\left(I R_{1}\right)=\ell\left(I R_{2}\right)$.

Proof. Since $\operatorname{rad}\left(J_{1}+J_{2}\right)=\operatorname{rad} J_{1}=\operatorname{rad} J_{2}$, it suffices to consider the case where $J_{1} \subseteq J_{2}$. With notation as in (2.2), $\operatorname{ker} \psi^{\prime}$ is a nilpotent ideal. Thus $\operatorname{dim} F_{R_{1}}\left(I R_{1}\right)=$ $\operatorname{dim} F_{R_{2}}\left(I R_{2}\right)$ and $\ell\left(I R_{1}\right)=\ell\left(I R_{2}\right)$.

As we remarked in Section 1, the dimension of the fiber cone $F(I)$ of an ideal $I$ is the same as the dimension of the fiber cone $F\left(I^{n}\right)$ of a power $I^{n}$ of $I$. Hence, with notation as in (2.1), we have $\operatorname{dim} F_{R^{\prime}}\left(I R^{\prime}\right)=\operatorname{dim} F_{R^{\prime}}\left(I^{n} R^{\prime}\right)$ and $\ell\left(I R^{\prime}\right)=\ell\left(I^{n} R^{\prime}\right)$ for each positive integer $n$.

\section{THE ASSOCIATED GRADED RING AND THE FIBER CONE.}

The associated graded ring of the ideal $I$ plays a role in the behavior of the fiber cone of the image of $I$ modulo a principal ideal as we illustrate in Proposition 3.1 and Example 3.2.

Proposition 3.1. Let $I \subseteq \mathbf{m}$ be an ideal of a Noetherian local ring $(R, \mathbf{m})$. For $x \in$ $\mathbf{m}$, let $x^{*}$ denote the image of $x$ in the associated graded ring $G(I)=R[I t] / I R[I t]$ and let $\bar{x}$ denote the image of $x$ in the fiber cone $F(I)$. If $x^{*}$ is a regular element of $G(I)$, then $F(I) / \bar{x} F(I) \cong F_{R^{\prime}}\left(I^{\prime}\right)$, where $R^{\prime}=R / x R$ and $I^{\prime}=I R^{\prime}$.

Proof. There exists a positive integer $s$ such that $x \in I^{s}-I^{s+1}$. Since $x^{*}$ is a regular element of $G(I)$ with $\operatorname{deg} x^{*}=s$, we have $\left(I^{n} \cap x R\right)=x I^{n-s}$ for every $n \geq 0$, where $I^{n-s}:=R$ if $n-s \leq 0$. Hence we have

$$
\left[\operatorname{ker} \psi_{x R}\right]_{n}=\frac{\left(I^{n} \cap x R\right)+\mathbf{m} I^{n}}{\mathbf{m} I^{n}}=\frac{x I^{n-s}+\mathbf{m} I^{n}}{\mathbf{m} I^{n}}=[\bar{x} F(I)]_{n},
$$

for every $n \geq 0$. Therefore $F(I) / \bar{x} F(I) \cong F_{R^{\prime}}\left(I^{\prime}\right)$.

With notation as in Proposition 3.1, the following example shows that for $x \in$ $I-\mathbf{m} I$ such that $\bar{x}$ is a regular element of $F(I)$, it may happen that $\bar{x} F(I) \subsetneq \operatorname{ker} \psi_{x R}$ and $F_{R^{\prime}}\left(I^{\prime}\right) \nRightarrow F_{R}(I) / \bar{x} F(I)$, where $R^{\prime}=R / x R$ and $I^{\prime}=I R^{\prime}$. Proposition 3.1 implies that for such an example $x^{*} \in G(I)$ is necessarily a zero divisor.

Example 3.2. Let $k$ be a field and consider the subring $R:=k\left[\left[t^{3}, t^{4}, t^{5}\right]\right]$ of the formal power series ring $k[[t]]$. Thus $R=k+t^{3} k[[t]]$ is a complete Cohen-Macaulay 
one-dimensional local domain. Let $I=\left(t^{3}, t^{4}\right) R$. An easy computation implies $I^{3}=t^{3} I^{2}$. Hence $t^{3} R$ is a principal reduction of $I$. Since $I$ is 2-generated, it follows from [DGH, Proposition 3.5] that $F(I)$ is Cohen-Macaulay and in fact a complete intersection. Let $X, Y$ be indeterminates over $k$ and define a $k$-algebra homomorphism $\phi: k[X, Y] \rightarrow F(I)$ by setting $\phi(X)=\overline{t^{3}}$ and $\phi(Y)=\overline{t^{4}}$. Then $\operatorname{ker} \phi=Y^{3} k[X, Y]$ and $F(I) \cong k[X, Y] / Y^{3} k[X, Y]$. Thus $\overline{t^{3}}$ is a regular element of $F(I)$ and $F(I) / \overline{t^{3}} F(I) \cong k[Y] / Y^{3} k[Y]$. Let $J=t^{3} R, R^{\prime}=R / J$ and $I^{\prime}=I R^{\prime}$. Since $t^{8} \in\left(I^{2} \cap J\right)$, we have $\phi\left(Y^{2}\right)=\overline{t^{8}} \in \operatorname{ker} \psi_{J}$ and $F_{R^{\prime}}\left(I^{\prime}\right) \cong k[Y] / Y^{2} k[Y]$. Thus $F(I) / \overline{t^{3}} F(I) \supsetneqq F_{R^{\prime}}\left(I^{\prime}\right)$. In fact, we have ker $\psi_{J}=\left(\overline{t^{3}}, \overline{t^{8}}\right) F(I)$ and $\overline{t^{8}} \notin \overline{t^{3}} F(I)$.

We list several observations and questions concerning the dimension of fiber cones and their behavior under homomorphic image.

Discussion 3.3. Let $I \subseteq \mathbf{m}$ be an ideal of a Noetherian local $\operatorname{ring}(R, \mathbf{m})$. If $J \subseteq \mathbf{m}$ is an ideal of $R$ and $R^{\prime}=R / J$, then there exists a surjective ring homomorphism $\chi_{J}: G_{R}(I)=R[I t] / I R[I t] \rightarrow G_{R^{\prime}}\left(I R^{\prime}\right)$ of the associated graded ring $G_{R}(I)$ of $I$ onto the associted graded ring $G_{R^{\prime}}\left(I R^{\prime}\right)$ of $I R^{\prime}$ [K, page 150].

We have the following commutative diagram involving the associated graded rings and fiber cones for which the vertical maps $\alpha$ and $\beta$ are surjective:

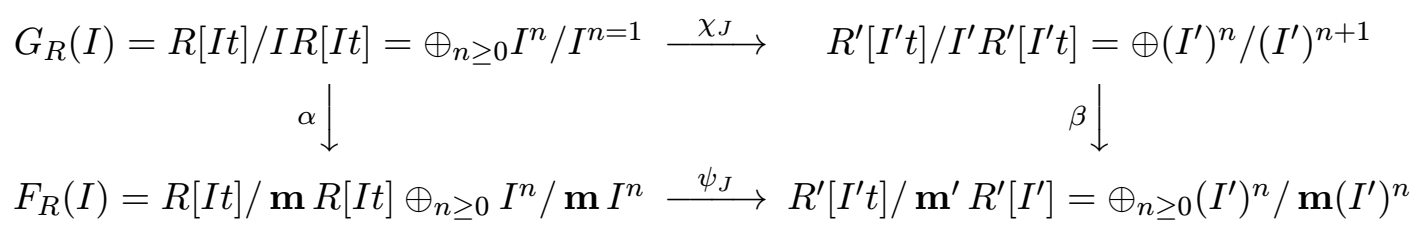

If $J$ is nonzero, then ker $\chi_{J} \neq 0$. It can happen, however, that $J$ is nonzero and yet ker $\psi_{J}=0$. This is possible even in the case where $I$ is $\mathbf{m}$-primary. In an example exhibiting this behavior, commutativity of the diagram above implies one must have $\operatorname{ker} \chi_{J} \subseteq \operatorname{ker} \alpha$.

Example 3.4. Let $k$ be a field and let $R=k[x, y]_{(x, y)}$, where $x^{2}=x y=0$. Let $I=y R$ and let $J=x R$. Then ker $\psi_{J}=\bigoplus_{n \geq 0} \frac{\left(x R \cap y^{n} R\right)+\mathbf{m} y^{n} R}{\mathbf{m} y^{n} R}=0$, but $J=x R \neq 0$.

A reason for the existence of examples such as Example 3.4 is given in Proposition 3.5.

Proposition 3.5. Suppose $(R, \mathbf{m})$ is a Noetherian local ring and $I$ is an $\mathbf{m}$-primary ideal. If the fiber cone $F(I)$ is an integral domain, then $\operatorname{ker} \psi_{J}=0$ for every ideal 
$J$ of $R$ such that $\operatorname{dim}(R / J)=\operatorname{dim} R$. In particular, if $I$ is $\mathbf{m}$-primary and $F(I)$ is an integral domain, then there exists a prime ideal $J$ of $R$ such that ker $\psi_{J}=0$.

Proof. Let $R^{\prime}:=R / J$. Since $I$ is $\mathbf{m}$-primary, $\operatorname{dim} F\left(I R^{\prime}\right)=\operatorname{dim} R^{\prime}$. Thus $\operatorname{dim} R^{\prime}=$ $\operatorname{dim} R$ implies $\operatorname{dim} F\left(I R^{\prime}\right)=\operatorname{dim} F(I)$. Since $F(I)$ is an integral domain, it follows that ker $\psi_{J}=0$. The last statement follows becaues there exists a prime ideal $J$ of $R$ such that $\operatorname{dim} R=\operatorname{dim}(R / J)$.

Propositon 3.5 and Example 3.4 show that with notation as in (3.1), it can happen that $x^{*} \in G(I)$ is not a regular element and yet $\operatorname{ker} \psi_{x R}=\bar{x} F(I)$.

In Section 4 we consider fiber cones $F(I)$ such that $\operatorname{ker} \psi_{J} \neq 0$ for each nonzero ideal $J$.

\section{MaXimal Fiber CONes With ReSPeCt to homomorphic image.}

Suppose $(R, \mathbf{m})$ is a Noetherian local ring and $I \subseteq \mathbf{m}$ is an ideal of $R$. If $J$ is a nonzero ideal of $R$ such that $\operatorname{ker} \psi_{J}=\bigoplus_{n \geq 0} \frac{\left(J \cap I^{n}\right)+\mathbf{m} I^{n}}{\mathbf{m} I^{n}}$ is the zero ideal of $F(I)$, then we have $F_{R}(I)=F_{R^{\prime}}\left(I R^{\prime}\right)$, where $R^{\prime}:=R / J$; so the fiber cone $F(I)$ is realized as a fiber cone of a proper homomorphic image $R^{\prime}$ of $R$. If there fails to exist such an ideal $J$, i.e., if ker $\psi_{J} \neq 0$ for each nonzero ideal $J$, then we say that $F(I)$ is a maximal fiber cone of $R$.

We record in Remark 4.1 some immediate consequences of the inequality $\operatorname{dim} F_{R^{\prime}}\left(I R^{\prime}\right) \leq$ $\operatorname{dim} R^{\prime}$.

Remark 4.1. With notation as in (2.1), we have:

(1) If $J$ is such that $\operatorname{dim} R^{\prime}<\operatorname{dim} R$ and if $\operatorname{dim} F_{R}(I)=\operatorname{dim} R$, then $\operatorname{ker} \psi_{J} \neq 0$.

(2) If $I$ is $\mathbf{m}$-primary and $J$ is not contained in a minimal prime of $R$, then $\operatorname{ker} \psi_{J} \neq 0$.

(3) If $R$ is an integral domain and $\operatorname{dim} F(I)=\operatorname{dim} R$, then $F(I)$ is a maximal fiber cone.

(4) If $R$ is an integral domain, then $F(I)$ is a maximal fiber cone for every m-primary ideal $I$ of $R$.

We are interested in describing all the maximal fiber cones of $R$. Thus we are interested in conditions on $I$ and $R$ in order that there exist a nonzero ideal $J$ of $R$ such that ker $\psi_{J}=0$. In considering this question, by Proposition 2.3, one may assume that $J=x R$ is a nonzero principal ideal. Thus the question can also be phrased: 
Question 4.2. Under what conditions on $I$ and $R$ does it follow for each nonzero element $x \in \mathbf{m}$ that $\operatorname{ker} \psi_{x R} \neq 0$ ?

Discussion 4.3. Information about Question 4.2 is provided by the work of Rees in [R]. In particular, [R, Theorem 2.1] implies that if $x \in \mathbf{m}$ is such that $\left(I^{n}: x\right)=I^{n}$ for each positive integer $n$, then $F_{R}(I)=F_{R^{\prime}}\left(I^{\prime}\right)$, where $R^{\prime}:=R / x R$ and $I^{\prime}:=I R^{\prime}$. Thus for $x \in \mathbf{m}$ a sufficient condition for $\operatorname{ker} \psi_{x R}=0$ is that $\left(I^{n}: x\right)=I^{n}$ for each positive integer $n$. It is readily seen that this colon condition on $x$ is equivalent to $x \notin I$ and the image of $x$ in the associated graded ring $G(I)=R[I t] / I R[I t]$ is a regular element. More generally, if $x \in I^{s}-I^{s+1}$ and if the image $x^{*}$ of $x$ in $G(I)$ is a regular element, then by Proposition $3.1 \operatorname{ker} \psi_{x R}=\bar{x} F(I)$. Thus if we also have $x \in \mathbf{m} I^{s}$, then ker $\psi_{x R}=0$. Example 3.4 shows that this sufficient condition for ker $\psi_{x R}=0$ is not a necessary condition.

Proposition 2.3 gives a necessary and sufficient condition on a principal ideal $J=x R$ in order that $\operatorname{ker} \psi_{x R}=0$, namely that $\left(I^{n}: x\right)=\left(\mathbf{m} I^{n}: x\right)$ for each integer $n \geq 0$. By Proposition 2.3, if ker $\psi_{x R}=0$, then also ker $\psi_{y x R}=0$ for every $y \in R$.

If $I=y R$ is a non-nilpotent principal ideal of $R$, we give in Corollary 4.5 necessary and sufficient conditions for $F(I)$ to be a maximal fiber cone.

Proposition 4.4. Suppose $(R, \mathbf{m})$ is a Noetherian local ring and $I=y R \subseteq \mathbf{m}$ is a non-nilpotent principal ideal of $R$. For $x \in \mathbf{m}$, we have $\operatorname{ker} \psi_{x R}=0 \Longleftrightarrow y^{n} \notin x R$ for each positive integer $n$.

Proof. We have ker $\psi_{x R}=0 \Longleftrightarrow\left(y^{n} R \cap x R\right) \subseteq \mathbf{m} y^{n} R$ for each positive integer $n$, and $y^{n} \notin x R \Longleftrightarrow\left(y^{n} R \cap x R\right) \subsetneq y^{n} R \Longleftrightarrow\left(y^{n} R \cap x R\right) \subseteq \mathbf{m} y^{n} R$.

Corollary 4.5. Let $(R, \mathbf{m})$ be a Noetherian local ring and $I=y R \subseteq \mathbf{m}$ be a nonnilpotent principal ideal of $R$. Then $F(I)$ is a maximal fiber cone if and only if $R$ is a one-dimensional integral domain.

Proof. By Proposition 4.4, for $x \in \mathbf{m}$ we have $y \in \operatorname{rad} x R \Longleftrightarrow \operatorname{ker} \psi_{x R} \neq 0$. Suppose $F(I)$ is a maximal fiber cone. Then by definition, $\operatorname{ker} \psi_{x R} \neq 0$ for each nonzero $x \in \mathbf{m}$. Since $y$ is not nilpotent, there exists a minimal prime $P$ of $R$ such that $y \notin P$. It follows that $P=0$, for if not, then there exists a nonzero $x \in P$ and $y \in \operatorname{rad} x R \subseteq P$ implies $y \in P$. Thus $R$ is an integral domain. Moreover, this same argument implies $y$ is in every nonzero prime of $R$. Since $R$ is Noetherian, it follows that $\operatorname{dim} R=1$. For $y R$ has only finitely many minimal primes and every minimal 
prime of $y R$ has height one by the Altitude Theorem of Krull [N, page 26] or [Mat, page 100]. If there exists $P \in \operatorname{Spec} R$ with ht $P>1$, then the Altitude Theorem of Krull implies $P$ is the union of the height-one primes contained in $P$. This implies there exist infinitely many height-one primes contained in $P$. Since $y$ is contained in only finitely many height-one primes, this is impossible. Thus $\operatorname{dim} R=1$. Since $R$ is local, $\mathbf{m}$ is the only nonzero prime of $R$.

Conversely, if $R$ is a one-dimensional Noetherian local integral domain, then (4.1) implies that $F(I)$ is a maximal fiber cone for every non-nilpotent principal ideal $I=y R \subseteq \mathbf{m}$.

Question 4.6. If $F(I)$ is a maximal fiber cone of $R$, does it follow that $\operatorname{dim} F(I)=$ $\operatorname{dim} R$ ?

Proposition 4.7. Suppose $(R, \mathbf{m})$ is a Noetherian local ring and $I \subseteq \mathbf{m}$ is an ideal of $R$. If $\operatorname{dim} F(I):=n=\operatorname{ht}(I)<\operatorname{dim} R$ and if $F(I)$ is an integral domain, then $F(I)$ is not a maximal fiber cone. In particular, if $I$ is of the principal class, i.e., $I=\left(a_{1}, \ldots, a_{n}\right) R$, where $\operatorname{ht}(I)=n$, and if $\operatorname{ht}(I)<\operatorname{dim} R$, then $F(I)$ is not a maximal fiber cone of $R$.

Proof. Choose $x \in \mathbf{m}$ such that $x$ is not in any minimal prime of $I$. Then $L:=$ $(I, x) R$ has height $n+1$. Let $\bar{x}$ denote the image of $x$ in the fiber cone $F_{R}(L)$. Then $F_{R}(L)$ is a homomorphic image of a polynomial ring in one variable $F_{R}(I)[z]$ over $F_{R}(I)$ by means of a homomorphism mapping $z \rightarrow \bar{x}$. Since $\operatorname{dim} F(I)=n$ and $F(I)$ is an integral domain, it follows that $F(I)[z] \cong F(L)$ by means of an isomorphism taking $z \rightarrow \bar{x}$. Let $J=x R$ and $R^{\prime}:=R / J$. Then $\operatorname{ht}\left(I R^{\prime}\right)=\operatorname{ht}(L / x R)=n$, so $\operatorname{dim} F_{R^{\prime}}\left(I R^{\prime}\right) \geq n$. Since $\psi_{J}: F_{R}(I) \rightarrow F_{R^{\prime}}\left(I R^{\prime}\right)$ is surjective and $F_{R}(I)$ is an $n$-dimensional integral domain, it follows that $\psi_{J}: F_{R}(I) \rightarrow F_{R^{\prime}}\left(I R^{\prime}\right)$ is an isomorphism. In particular, if $I$ is of the principal class, then $F(I)$ is a polynomial ring in $n$ variables over the field $R / \mathbf{m}$, so $F(I)$ is an integral domain with $\operatorname{dim} F(I)=$ $\operatorname{ht}(I)$.

If $I$ is generated by a regular sequence, then $I$ is of the principal class. Thus if $F(I)$ is a maximal fiber cone and $I$ is generated by a regular sequence, then by Proposition 4.7, $\operatorname{dim} F(I)=\operatorname{dim} R$.

We observe in Proposition 4.8 a situation where the integral domain hypothesis of Proposition 4.7 applies.

Proposition 4.8. Let $A=k\left[X_{1}, X_{2}, \cdots, X_{d}\right]=\bigoplus_{n=0}^{\infty} A_{n}$ be a polynomial ring in $d$ variables over a field $k$ and let $\mathbf{m}=\left(X_{1}, X_{2}, \cdots, X_{d}\right) A$ denote its homogeneous 
maximal ideal. Suppose $I=\left(f_{1}, f_{2}, \cdots, f_{n}\right) A$, where $f_{1}, f_{2}, \cdots, f_{n}$ are homogeneous polynomials all of the same degree $t$. Let $R=A_{\mathbf{m}}$. Then $F(I R)$ is an integral domain. Thus if $F(I R)$ is a maximal fiber cone, then $\operatorname{dim} F(I R)=d$.

Proof. We have

$$
k\left[f_{1}, f_{2}, \cdots, f_{n}\right]=k \oplus I_{1} \oplus I_{2} \oplus \cdots,
$$

where $I_{i}=I^{i} \cap A_{i t}$ for $i>0$. Since $I^{i} / \mathbf{m} I^{i} \cong I^{i} \cap A_{i t}$ for $i \geq 0$, we have the following isomorphisms:

$$
k\left[f_{1}, f_{2}, \cdots, f_{n}\right] \cong \oplus_{i=0}^{\infty}\left(I^{i} / \mathbf{m} I^{i}\right) \cong \oplus_{i=0}^{\infty}\left(I^{i} R / \mathbf{m} I^{i} R\right)=F(I R) .
$$

Therefore $F(I R)$ is an integral domain. The result now follows from Proposition 4.7 .

Corollary 4.9. With notation as in Proposition 4.8, if $\operatorname{dim} F(I)=\operatorname{ht} I$ and $F(I R)$ is a maximal fiber cone, then I is $\mathbf{m}$-primary.

Proof. We have $\operatorname{dim} F(I R)=d$ by Proposition 4.8. Since $I$ is homogeneous ideal and ht $I=d, \mathbf{m}$ is the unique homogeneous minimal prime of $I$, Therefore $I$ is m-primary.

Question 4.10. Let $(R, \mathbf{m})$ be a Noetherian local ring and let $I \subseteq \mathbf{m}$ be an ideal of $R$. If $\operatorname{dim} F(I)=$ ht $I$ and $F(I)$ is a maximal fiber cone, does it follow that $I$ is m-primary?

Remark 4.11. Without the assumption in Question 4.10 that $\operatorname{dim} F(I)=\mathrm{ht} I$, it is easy to give examples where $F(I)$ is a maximal fiber cone and yet $I$ is not $\mathbf{m}$-primary. For example, with notation as in Proposition 4.8, if $d>1$ and $I=$ $\left(X_{1}^{2}, X_{1} X_{2}, \ldots, X_{1} X_{d}\right) A$, then $\operatorname{ht}(I R)=1$, but $\operatorname{dim} F(I R)=d$ and $F(I R)$ is a maximal fiber cone.

\section{When IS THE FIBER CONE A HYPERSURFACE?}

Setting 5.1. Let $I \subseteq \mathbf{m}$ be an ideal of a Noetherian local ring $(R, \mathbf{m})$. In this section we consider the structure of the fiber cone $F(I)=\oplus_{n \geq 0} F_{n}$ in the case where $\operatorname{dim} F(I)=d>0$ and $\mu(I)=d+1$. If $a_{1}, \ldots, a_{d+1}$ is a basis for $F_{1}=$ $I / \mathbf{m} I$ as a vector space over the field $k:=R / \mathbf{m}$, then there exists a presentation $\phi: k\left[X_{1}, \ldots, X_{d+1}\right] \rightarrow F(I)$ of $F(I)$ as a graded $k$-algebra homomorphic image of a polynomial ring in $d+1$ variables over $k$ defined by setting $\phi\left(X_{i}\right)=a_{i}$, for $i=1, \ldots, d+1$. Moreover, $F(I)$ is a hypersurface if and only if $\operatorname{ker} \phi$ is a principal ideal [K, Examples 1.2]. 
Lemma 5.2. Let $(R, \mathbf{m})$ be a Noetherian local ring having infinite residue field $R / \mathbf{m}:=k$, and let $I \subseteq \mathbf{m}$ be an ideal of $R$ such that $\operatorname{dim} F(I)=d>0$ and $\mu(I)=$ $d+1$. Let $r=r(I)$ denote the reduction number of $I$ and let $\phi: k\left[X_{1}, \ldots, X_{d+1}\right] \rightarrow$ $F(I)$ be a presentation of the fiber cone $F(I)$ as in Setting 5.1. Then the minimal degree of a nonzero form $f \in \operatorname{ker} \phi$ is $r+1$.

Proof. The map $\phi$ from the graded ring $A=k\left[X_{1}, \ldots, X_{d+1}\right]=\oplus_{n \geq 0} A_{n}$ onto the graded ring $F(I)=\oplus_{n \geq 0} F_{n}=\oplus_{n \geq 0}\left(I^{n} / \mathbf{m} I^{n}\right)$ is a surjective graded $k$-algebra homomorphism of degree 0 . Let $K:=\operatorname{ker} \phi=\oplus_{n \geq 0} K_{n}$. For each positive integer $n$ we have a short exact sequence

$$
0 \rightarrow K_{n} \rightarrow A_{n} \rightarrow F_{n} \rightarrow 0
$$

of finite-dimensional vector spaces over $k$. Since $I$ has reduction number $r$, it follows from [ES, Theorem, page 440] that $\operatorname{dim}_{k} F_{i}=\mu\left(I^{i}\right)=\left(\begin{array}{c}i+d \\ d\end{array}\right)$ for $i=0,1, \ldots, r$ and $\operatorname{dim}_{k} F_{r+1}=\mu\left(I^{r+1}\right)<\left(\begin{array}{c}r+d+1 \\ d\end{array}\right)$. Since $\operatorname{dim} A_{i}=\left(\begin{array}{c}i+d \\ d\end{array}\right)$ for all $i$, it follows that $K_{i}=0$ for $i=0, \ldots, r$ and $K_{r+1} \neq 0$. Hence the minimal degree of a nonzero form $f \in \operatorname{ker} \phi$ is $r+1$.

Remark 5.3. Let $A=k\left[X_{1}, \ldots, X_{n}\right]$ be a polynomial ring in $n$ variables $X_{1}, \ldots, X_{n}$ over a field $k$. For an ideal $K$ of $A$, it is well known that $\operatorname{ht}(K)=1$ if and only if $\operatorname{dim}(A / K)=n-1[\mathrm{~K}$, Corollary 3.6, page 53]. Moreover, $K$ is principal if and only if $\operatorname{ht}(P)=1$ for each associated prime $P$ of $K$. If $K=\left(g_{1}, \ldots, g_{m}\right) A$ and $g$ is a greatest common divisor of $g_{1}, \ldots, g_{m}$, then $K=g J$, where $\operatorname{ht}(J)>1$. Thus $K$ is principal if and only if $J=A$. If $K$ is homogeneous, then $g_{1}, \ldots, g_{m}$ may be taken to be homogeneous; it then follows that $g$ is homogeneous and $K=g J$, where $J$ is homogeneous with ht $(J)>1$. If $K=\operatorname{rad} K$, then each associated prime of $K$ is a minimal prime and $K$ is principal if and only if $h t(P)=1$ for each minimal prime $P$ of $K$.

Proposition 5.4. Let $(R, \mathbf{m})$ be a Noetherian local ring with infinite residue field $k=R / \mathbf{m}$ and let $I \subseteq \mathbf{m}$ be an ideal of $R$ such that $\operatorname{dim} F(I)=d>0$ and $\mu(I)=$ $d+1$. Let $\phi: A=k\left[X_{1}, \ldots, X_{d+1}\right] \rightarrow F(I)$ be a presentation of $F(I)$ as a graded homomorphic image of a polynomial ring as in Setting 5.1. Let $f \in K:=\operatorname{ker} \phi$ be a nonzero homogeneous form of minimal degree. Then the following are equivalent.

(1) $\operatorname{ker} \phi=f A$, i.e., $F(I)$ is a hypersurface.

(2) ht $P=1$ for each $P \in$ Ass $K$.

(3) $F(I)$ is a Cohen-Macaulay ring.

(4) $\operatorname{deg} f=e(F(I))$, the multiplicity of $F(I)$. 
Proof. That (1) is equivalent to (2) is observed in Remark 5.2. It is clear that (1) implies (3) and it follows from [BH, (2.2.15) and (2.1.14)] that (3) implies (2). To see the equivalence of (3) and (4), we use [DRV, Theorem 2.1]. By Lemma 5.2, $\operatorname{deg} f=r+1$, where $r$ is the reduction number of $I$.

Since $\operatorname{dim} F(I)=d$, there exists a minimal reduction $J=\left(x_{1}, \ldots, x_{d}\right) R$ of $I$ and $y \in I$ such that $I=J+y R$. By [DRV, Theorem 2.1], $F(I)$ is Cohen-Macaulay if and only if

$$
e(F(I))=\sum_{n=0}^{r} \lambda\left(\frac{I^{n}}{J I^{n-1}+\mathbf{m} I^{n}}\right) .
$$

Since for $0 \leq n \leq r, \lambda\left(\frac{I^{n}}{J I^{n-1}+\mathbf{m} I^{n}}\right)=1$, the sum on the right hand side of the displayed equation is $r+1=\operatorname{deg} f$. This proves the equivalence of (3) and (4).

Remark 5.5. With notation as in Proposition 5.4, we have the following inequality $e(F(I)) \leq \operatorname{deg} f$, where $e(F(I))$ is the multiplicity of $F(I)$. Hence by Proposition 5.4, $F(I)$ is not Cohen-Macaulay $\Longleftrightarrow e(F(I))<\operatorname{deg} f$.

Proof. Let $J=\left(x_{1}, \ldots, x_{d}\right) R$ be a minimal reduction of $I$. Then $J F(I)$ is generated by a homogeneous system of parameters for $F(I)$ and

$$
\lambda\left(\frac{F(I)}{J F(I)}\right)=\sum_{n=0}^{r} \lambda\left(\frac{I^{n}}{J I^{n-1}+\mathbf{m} I^{n}}\right) .
$$

Let $\mathcal{M}$ denote the maximal homogeneous ideal of $F(I)$. Then

$$
e(F(I))=e\left(F(I)_{\mathcal{M}}\right) \leq \lambda\left(\frac{F(I)_{\mathcal{M}}}{J F(I)_{\mathcal{M}}}\right)=\lambda\left(\frac{F(I)}{J F(I)}\right) .
$$

Thus $e(F(I)) \leq \operatorname{deg} f=r+1$. Hence by Proposition 5.4, $F(I)$ is not CohenMacaulay if and only if $e(F(I))<\operatorname{deg} f$.

Theorem 5.6. Let $(R, \mathbf{m})$ be a Noetherian local ring with infinite residue field $k=R / \mathbf{m}$ and let $I \subseteq \mathbf{m}$ be an ideal of $R$ such that $\operatorname{dim} F(I)=d>0$ and $\mu(I)=d+1$. Suppose there exists a minimal reduction $J$ of $I$ generated by a regular sequence. Assume that grade $\left(G_{+}(I)\right) \geq d-1$. Then $F(I)$ is Cohen-Macaulay and thus a hypersurface.

Proof. For $x \in R$, let $x^{*}$ denote the image of $x$ in $G(I)$ and let $\bar{x}$ denote the image of $x$ in $F(I)$. There exists a minimal reduction $J=\left(x_{1}, \ldots, x_{d}\right) \subseteq I$ and $x_{d+1} \in I$ such that

(I) $\left\{x_{1}, \ldots, x_{d}\right\}$ is a regular sequence in $R$.

(II) $\left\{x_{1}, \ldots, x_{d}, x_{d+1}\right\}$ is a minimal set of generators of $I$.

(III) $\left\{x_{1}^{*}, \ldots, x_{d-1}^{*}\right\}$ is a regular sequence in $G(I)$. 
Let $R^{\prime}=R /\left(x_{1}, \ldots, x_{d-1}\right) R$, let $\mathbf{m}^{\prime}=\mathbf{m} /\left(x_{1}, \ldots, x_{d-1}\right) R$ and let $I^{\prime}=I R^{\prime}$. By Condition II, $I^{\prime}$ is a 2-generated ideal having a principal reduction generated by the image $x_{d}^{\prime}$ of $x_{d}$. Condition I implies that $x_{d}^{\prime}$ is a regular element of $R^{\prime}$. Hence by [DGH, Proposition 3.5], $F_{R^{\prime}}\left(I^{\prime}\right)$ is Cohen-Macaulay.

As observed in (2.1), the kernel of the canonical map $\psi: F_{R}(I) \rightarrow F_{R^{\prime}}\left(I^{\prime}\right)$ is

$$
\oplus_{n \geq 0} \frac{\left(I^{n} \cap\left(x_{1}, \ldots, x_{d-1}\right)\right)+\mathbf{m} I^{n}}{\mathbf{m} I^{n}} .
$$

Condition III and Proposition 3.1 imply

$$
\operatorname{ker} \psi=\oplus_{n \geq 0} \frac{\left(x_{1}, \ldots, x_{d-1}\right) I^{n-1}+\mathbf{m} I^{n}}{\mathbf{m} I^{n}}=\left(\overline{x_{1}}, \ldots, \overline{x_{d-1}}\right) F(I) .
$$

Hence

$$
\frac{F(I)}{\left(\overline{x_{1}}, \ldots, \overline{x_{d-1}}\right)} \cong F_{R^{\prime}}\left(I^{\prime}\right)
$$

and to show $F(I)$ is Cohen-Macaulay, it suffices to show $\left\{\overline{x_{1}}, \ldots, \overline{x_{d-1}}\right\}$ is a regular sequence in $F(I)$. By the generalized Vallabrega-Valla criterion of Cortadellas and Zarzuela [CZ, Theorem 2.8], to show $\left\{\overline{x_{1}}, \ldots, \overline{x_{d-1}}\right\}$ is a regular sequence in $F(I)$, it suffices to show

$$
\left(x_{1}, \ldots, x_{d-1}\right) \cap \mathbf{m} I^{n+1}=\left(x_{1}, \ldots, x_{d-1}\right) \mathbf{m} I^{n}, \text { for all } n \geq 0 .
$$

" $\supseteq$ " is clear. We prove " $\subseteq$ " by induction on $n$.

(Case i ) $n=0$ : Let $u \in\left(x_{1}, \ldots, x_{d-1}\right) \cap \mathbf{m} I$. Thus $u=\sum_{i=1}^{d-1} r_{i} x_{i}=\sum_{j=1}^{d+1} \alpha_{j} x_{j}$, where $r_{i} \in R$ and $\alpha_{j} \in \mathbf{m}$. Therefore

$$
\left(r_{1}-\alpha_{1}\right) x_{1}+\cdots+\left(r_{d-1}-\alpha_{d-1}\right) x_{d-1}-\alpha_{d} x_{d}-\alpha_{d+1} x_{d+1}=0 .
$$

Since $\left\{x_{1}, \ldots, x_{d+1}\right\}$ is a minimal generating set for $I$, each $r_{i}-\alpha_{i} \in \mathbf{m}$. Since $\alpha_{i} \in \mathbf{m}, r_{i} \in \mathbf{m}$. Hence $u=\sum_{i=1}^{d-1} r_{i} x_{i} \in \mathbf{m}\left(x_{1}, \ldots, x_{d-1}\right)$.

(Case ii ) $1 \leq n<r$, where $r=r_{J}(I)$ is the reduction number of $I$ with respect to $J$ : We have $\left(x_{1}, \ldots, x_{d-1}\right) \cap \mathbf{m} I^{n+1}=\left(x_{1}, \ldots, x_{d-1}\right) \cap\left(I^{n+1} \cap \mathbf{m} I^{n+1}\right)$ $=\left(\left(x_{1}, \ldots, x_{d-1}\right) \cap I^{n+1}\right) \cap \mathbf{m} I^{n+1}=\left(\left(x_{1}, \ldots, x_{d-1}\right) I^{n} \cap \mathbf{m} I^{n+1}\right.$, the last equality by Condition III.

Hence $u \in\left(x_{1}, \ldots, x_{d-1}\right) \cap \mathbf{m} I^{n+1}$ implies $u \in\left(\left(x_{1}, \ldots, x_{d-1}\right) I^{n} \cap \mathbf{m} I^{n+1}\right.$. Thus $u=\sum_{i=1}^{d-1} x_{i} g_{i}$, where $g_{i} \in I^{n}$ and $u=H\left(x_{1}, \ldots, x_{d+1}\right)$, where $H\left(X_{1}, \ldots, X_{d+1}\right) \in$ $R\left[X_{1}, \ldots, X_{d+1}\right]$ is a homogeneous polynomial with coefficients in $\mathbf{m}$ of degree $n+1$. Let $G_{i}\left(X_{1}, \ldots, X_{d+1}\right) \in R\left[X_{1}, \ldots, X_{d+1}\right]$ be a homogeneous polynomial of degree $n$ such that $G_{i}\left(x_{1}, \ldots, x_{d+1}\right)=g_{i}$.

Let $\tau: R\left[X_{1}, \ldots, X_{d+1}\right] \rightarrow R[I t]$, where $\tau\left(X_{i}\right)=x_{i} t$ be a presentation of the Rees algebra $R[I t]$. Consider the following commutative diagram. 


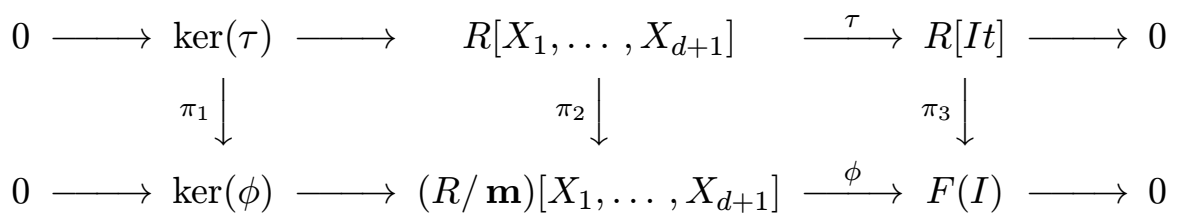

Since $\sum_{i=1}^{d-1} x_{i} g_{i}-H\left(x_{1}, \ldots, x_{d+1}\right)=0$, the homogeneous polynomial

$$
\sum_{i=1}^{d-1} X_{i} G_{i}\left(X_{1}, \ldots, X_{d+1}\right)-H\left(X_{1}, \ldots, X_{d+1}\right) \in \operatorname{ker} \tau .
$$

Since $H\left(X_{1}, \ldots, X_{d+1}\right)$ has coefficients in $\mathbf{m}$, we have

$$
0=\pi_{3} \tau\left(\sum_{i=1}^{d-1} X_{i} G_{i}-H\right)=\phi \pi_{2}\left(\sum_{i=1}^{d-1} X_{i} G_{i}-H\right)=\phi \pi_{2}\left(\sum_{i=1}^{d-1} X_{i} G_{i}\right) .
$$

Hence $\pi_{2}\left(\sum_{i=1}^{d-1} X_{i} G_{i}\right) \in \operatorname{ker} \phi$. Since $\sum_{i=1}^{d-1} X_{i} G_{i}$ is of degree $n+1 \leq r$, Lemma 5.2 implies $\pi_{2}\left(\sum_{i=1}^{d-1} X_{i} G_{i}\right)=0$. Therefore the coefficients of $\sum_{i=1}^{d-1} X_{i} G_{i}$ are in m. Evaluating this polynomial by mapping $X_{i} \mapsto x_{i}$ gives $u=\sum_{i=1}^{d-1} x_{i} g_{i} \in$ $\left(x_{1}, \ldots, x_{d_{1}}\right) \mathbf{m} I^{n}$.

( Case iii ) $n \geq r$ : Since $n \geq r$, we have $I^{n+1}=J I^{n}=\left(x_{1}, \ldots, x_{d}\right) I^{n}$.

Let $u \in\left(x_{1}, \ldots, x_{d-1}\right) \cap \mathbf{m} I^{n+1}=\left(x_{1}, \ldots, x_{d-1}\right) \cap \mathbf{m}\left(x_{1}, \ldots, x_{d}\right) I^{n}$. Thus $u=$ $\sum_{i=1}^{d-1} r_{i} x_{i}=\sum_{j=1}^{d} \alpha_{j} x_{j}$, where each $r_{i} \in R$ and each $\alpha_{j} \in \mathbf{m} I^{n}$. Hence $\alpha_{d} x_{d}=$ $\sum_{i=1}^{d-1}\left(r_{i}-\alpha_{i}\right) x_{i}$ and this implies $\alpha_{d} \in\left(\left(x_{1}, \ldots, x_{d-1}\right): x_{d}\right)=\left(x_{1}, \ldots, x_{d-1}\right)$, the last equality because of Condition I. Hence

$$
\alpha_{d} \in\left(x_{1}, \ldots, x_{d-1}\right) \cap \mathbf{m} I^{n}=\left(x_{1}, \ldots, x_{d-1}\right) \mathbf{m} I^{n-1},
$$

the last equality because of our inductive hypothesis. Thus $u=\sum_{j=1}^{d} \alpha_{j} x_{j}=$ $\sum_{j=1}^{d-1} \alpha_{j} x_{j}+\alpha_{d} x_{d} \in\left(x_{1}, \ldots, x_{d-1}\right) \mathbf{m} I^{n}+\left(x_{1}, \ldots, x_{d-1}\right) \mathbf{m} I^{n-1} I=\left(x_{1}, \ldots, x_{d-1}\right) \mathbf{m} I^{n}$. This completes the proof that $\left\{\overline{x_{1}}, \ldots, \overline{x_{d-1}}\right\}$ is a regular sequence in $F(I)$, and thus the proof of Theorem 5.6

\section{The Cohen-Macaulay property of one-Dimensional fiber cones}

We record in this short section several consequences of a result of D'Cruz, Raghavan and Verma [DRV, Theorem 2.1] for the Cohen-Macaulay property of the fiber cone of a regular ideal having a principal reduction.

Proposition 6.1. Let $(R, \mathbf{m})$ be a Noetherian local ring and let $I \subseteq \mathbf{m}$ be a regular ideal having a principal reduction aR. Let $r=r_{a R}(I)$ be the reduction number of $I$ with respect to aR. Then the following are equivalent. 
(1) $F(I)$ is a Cohen-Macaulay ring.

(2) $\lambda\left(\frac{a I^{n}+\mathbf{m} I^{n+1}}{\mathbf{m} I^{n+1}}\right)=\lambda\left(\frac{I^{n}}{\mathbf{m} I^{n}}\right) \quad$ for $1 \leq n \leq r-1$.

Proof. $(1) \Rightarrow(2)$. Suppose $F(I)$ is a Cohen-Macaulay ring. Then $\bar{a}(=a+\mathbf{m} I)$ is a regular element of $F(I)$ with $\operatorname{deg} \bar{a}=1$. Let $F(I)=\oplus_{n \geq 0} F_{n}$, where $F_{n}=I^{n} / \mathbf{m} I^{n}$, and consider the graded $k$-algebra homomorphism $\phi_{\bar{a}}: F_{n} \rightarrow F_{n+1}$ given by $\phi_{\bar{a}}(\bar{x})=$ $\bar{x} \cdot \bar{a}$, for every $\bar{x} \in F_{n}$. Since $\bar{a}$ is a regular element of $F(I), \operatorname{dim}_{k} F_{n}=\operatorname{dim}_{k}\left(\bar{a} F_{n}\right)$.

For $1 \leq n \leq r-1$, we have

$$
\lambda\left(\frac{a I^{n}+\mathbf{m} I^{n+1}}{\mathbf{m} I^{n+1}}\right)=\lambda\left(\bar{a}\left(\frac{I^{n}}{\mathbf{m} I^{n}}\right)\right)=\operatorname{dim}_{k}\left(\bar{a} F_{n}\right)=\operatorname{dim}_{k}\left(F_{n}\right)=\lambda\left(\frac{I^{n}}{\mathbf{m} I^{n}}\right) .
$$

$(2) \Rightarrow(1)$. Suppose that $\lambda\left(\frac{a I^{n}+\mathbf{m} I^{n+1}}{\mathbf{m} I^{n+1}}\right)=\lambda\left(\frac{I^{n}}{\mathbf{m} I^{n}}\right)$, for $1 \leq n \leq r-1$. Since $a$ is a non-zero-divisor $R, I^{n+r} / \mathbf{m} I^{n+r} \cong I^{r} / \mathbf{m} I^{r}$, for every $n \geq 1$. Hence $e(F(I))=\lambda\left(I^{r} / \mathbf{m} I^{r}\right)$. To see the Cohen-Macaulay property of $F(I)$, we use [DRV, Theorem 2.1]. We have the following:

$$
\begin{aligned}
\sum_{n=0}^{r} \lambda\left(\frac{I^{n}}{a I^{n-1}+\mathbf{m} I^{n}}\right) & =\lambda\left(\frac{R}{\mathbf{m}}\right)+\sum_{n=1}^{r} \lambda\left(\frac{I^{n}}{a I^{n-1}+\mathbf{m} I^{n}}\right) \\
& =\lambda\left(\frac{R}{\mathbf{m}}\right)+\sum_{n=1}^{r}\left[\lambda\left(\frac{I^{n}}{\mathbf{m} I^{n}}\right)-\lambda\left(\frac{a I^{n-1}+\mathbf{m} I^{n}}{\mathbf{m} I^{n}}\right)\right] \\
& =\lambda\left(\frac{R}{\mathbf{m}}\right)+\sum_{n=1}^{r}\left[\lambda\left(\frac{I^{n}}{\mathbf{m} I^{n}}\right)-\lambda\left(\frac{I^{n-1}}{\mathbf{m} I^{n-1}}\right)\right] \\
& =\lambda\left(\frac{I^{r}}{\mathbf{m} I^{r}}\right) \\
& =e(F(I)) .
\end{aligned}
$$

Hence by [DRV, Theorem 2.1], $F(I)$ is a Cohen-Macaulay ring.

As an immediate consequence of Proposition 6.1 we have

Corollary 6.2. Let $(R, \mathbf{m})$ be a Noetherian local ring and $I$ be a regular ideal having a principal reduction aR with $r_{a R}(I)=2$. If $\mu(I)=n$, then

$$
F(I) \text { is Cohen-Macaulay } \Longleftrightarrow \lambda\left(\frac{a I+\mathbf{m} I^{2}}{\mathbf{m} I^{2}}\right)=n .
$$

Example 6.3 shows that Proposition 6.1 and Corollary 6.2 may fail to be true without the assumption on the length of $\frac{a I^{n}+\mathbf{m} I^{n+1}}{\mathbf{m} I^{n+1}}$.

Example 6.3. Let $k$ be a field and consider the subring $R=k\left[\left[t^{3}, t^{7}, t^{11}\right]\right]$ of the formal power series ring $k[[t]]$. Let $I=\left(t^{6}, t^{7}, t^{11}\right) R$. An easy computation implies $t^{6} I \neq I^{2}$ and $t^{6} I^{2}=I^{3}$. Hence $r_{t^{6} R}(I)=2$. Note that $\overline{t^{6}} F(I)$ is a homogeneous system of parameter of $F(I)$. But $\overline{t^{6} t^{11}}=\left(t^{6}+\mathbf{m} I\right)\left(t^{11}+\mathbf{m} I\right)=t^{17}+\mathbf{m} I^{2}=$ 
0 , and hence $F(I)$ is not a Cohen-Macaulay ring. And $\lambda\left(\frac{t^{6} I+\mathbf{m} I^{2}}{\mathbf{m} I^{2}}\right)=\lambda\left(\frac{I^{2}}{\mathbf{m} I^{2}}\right)-$ $\lambda\left(\frac{I^{2}}{t^{6} I+\mathbf{m} I^{2}}\right)=2<3$.

\section{REFERENCES}

[AM] M. F. Atiyah and I. G. Macdonald, Introduction to Commutative Algebra, Addison-Wesley, London, 1969.

[BH] W. Bruns and J. Herzog, Cohen-Macaulay Rings, Cambridge University Press, Cambridge, 1993.

[CGPU] A. Corso, L. Ghezzi, C. Polini and B. Ulrich, Cohen-Macaulayness of special fiber rings, in preparation.

[CZ] T. Cortadellas and S. Zarzuela, On the depth of the fiber cone of filtrations, J. Algebra 198 (1997), 428-445.

[DGH] M. D'Anna, A. Guerrieri and W. Heinzer, Ideals having a one-dimensional fiber cone, Ideal Theoretic Methods in Commutative Algebra, Marcel Dekker, 2001, pp. 155-170.

[DRV] C. D'Cruz, K.N. Raghavan and J. Verma, Cohen-Macaulay fiber cone, Commutative Algebra, Algebraic Geometry and computational methods, (Hanoi 1996), Springer, Singapore 1999, pp. 233-246.

[ES] P. Eakin and A. Sathaye, Prestable ideals, J. Algebra 41 (1976), 439-454.

$[\mathrm{Hu}] \quad$ S. Huckaba, Analytic spread modulo an element and symbolic Rees algebras, J. Algebra 128 (1990), 306-320.

[K] E. Kunz, Introduction to Commutative Algebra and Algebraic Geometry, Birkhauser, Bosten, 1985.

[Mat] H. Matsumura, Commutative ring theory, Cambridge Univ. Press, Cambridge, 1986.

[N] M. Nagata, Local Rings, Interscience, New York, 1962.

[NR] D.G. Northcott and D. Rees, Reductions of ideals in local rings, Proc. Camb. Phil. Soc. 50 (1954), 145-158.

[R] D. Rees, Rings associted with ideals and analytic spread, Math. Proc. Camb. Phil. Soc. 89 (1981), 423-432. 